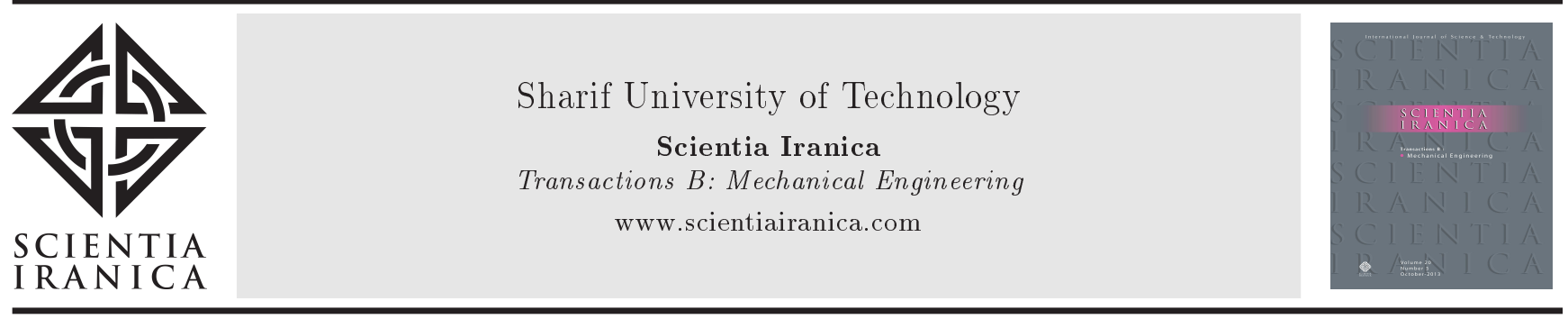

\title{
Algorithm development for aerodynamic preliminary design of multi-stage axial compressors
}

\author{
R. Azizi ${ }^{\mathrm{a}, *}$, R. Ebrahimi ${ }^{\mathrm{a}}$ and M. Ziabasharhagh ${ }^{\mathrm{b}}$ \\ a. Faculty of Aerospace Engineering, K. N. Toosi University, Tehran, P.O. Box 16765-3381, Iran. \\ b. Faculty of Mechanical Engineering, K. N. Toosi University, Tehran, P.O. Box 1999-19395, Iran. \\ Received 12 April 2015; received in revised form 23 August 2015; accepted 28 September 2015
}

\section{KEYWORDS \\ Axial compressor; Aerodynamic preliminary design; \\ Velocity triangles \\ determination; \\ Annulus sizing; \\ Blade design.}

\begin{abstract}
An algorithm for aerodynamic preliminary design of axial compressors has been developed. The aerodynamic design modules are meanline design and annulus sizing, velocity triangle determination, blade design, and axial compressor three-dimensional geometry generator. The hub and tip radii are computed through the meanline method and the velocity triangles are determined through radial equilibrium and vortex equations. Using incidence and deviation angles correlations of NACA 65, DCA, and NACA 63A4K6 profiles, the blades sections are designed on the compressor streamlines. The three-dimensional geometry of the designed compressors is the output of the developed preliminary design algorithm, which is generated through the developed geometrical code. This code generates the profile coordinates of blades sections and stacks them over a guideline curve and assembles them on the compressor axis. In different sections of this article, the aforementioned modules are explained, the developed algorithm is presented, the required design variables are introduced, and the design constraints are investigated. Finally, the aero-dynamical and geometrical specifications of the redesigned NACA 8-stage compressor achieved through the developed algorithm are presented and compared with the ones from original compressor.
\end{abstract}

(C) 2016 Sharif University of Technology. All rights reserved.

\section{Introduction}

The axial compressor design, the process of which is illustrated in Figure 1 [1-3], is based on some overall characteristics including mass flow, pressure ratio, and minimum required efficiency, which are determined in the gas turbine cycle analysis. In addition, designers try to obtain a compressor with maximum surge margin and minimum weight and dimension. Designing the compressor is composed of preliminary and detailed design steps. In the preliminary design, many features of the compressor such as stage number, rpm, blade

*. Corresponding author. Tel.: +98 21 r7339794

E-mail addresses: roholahazizi@dena.kntu.ac.ir (R. Azizi); rebrahimi@kntu.ac.ir (R. Ebrahimi); mzia@kntu.ac.ir (M. Ziabasharhagh) loading, annulus size, and number of blades are determined and the initial three-dimensional geometry of the blades is also constructed. The preliminary design is broken down into three steps of "aerodynamic design", "performance curve prediction", and "mechanical analysis". The compressor preliminary design is done based on a set of design variables values. The aero-dynamical and geometrical features of the axial compressor are generated in the aerodynamic design step. Some or all of the design variables might be modified, provided that the compressor performance curves, which are derived in the performance prediction step, do not satisfy the predetermined overall design performance (i.e., mass flow, pressure ratio, surge margin, or efficiency). Modification might also be essential in case of excessive stress or unsuitable blades frequencies, which are obtained in the mechanical analysis step. 


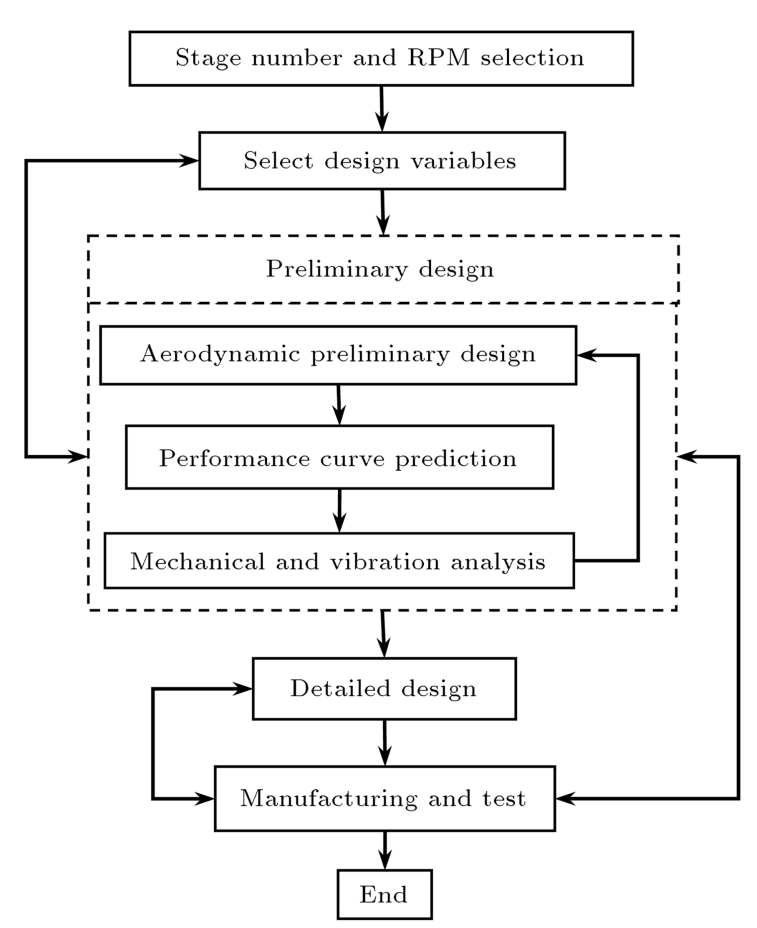

Figure 1. Axial compressor design steps.

In the detailed design step, the preliminary compressor is used as the input and some of its specifications are modified through Computational Fluid Dynamic (CFD) and optimization tools [4,5]. In the detailed design, many of the preliminary design features are considered fixed and this leads to high dependency of the final design on the preliminary one. In fact, it should not be expected that a compressor with good performance characteristics would be obtained in the detailed design step if its aerodynamic preliminary design is far away from these characteristics. For this reason, the first step in the development of a complete axial compressor design software is the development of an accurate aerodynamic preliminary design algorithm. The axial compressor aerodynamic preliminary design is done by the conventional design methods (i.e. meanline and streamline curvature methods) and conventional blade profiles (i.e. NACA63-A4K6, NACA 65, DCA, and MCA). Different industrial axial compressors have applied these methods and profiles in their preliminary or final design. The axial compressors of M7A-03 8MW gas turbine [6], LM25001 General Electric gas turbine [7], and KHI 20MW gas turbine [8] are some examples.

As can be seen in Figure 1, the Aerodynamic Preliminary Design (APD) should be combined with a performance simulator, the combination of which is named Aerodynamic Preliminary Design and Simulation (APDS) algorithm, as shown in Figure 2. The algorithm starts by the selection of some inputs which are categorized into two groups of overall inputs, comprised of the compressor mass flow, rpm, stage

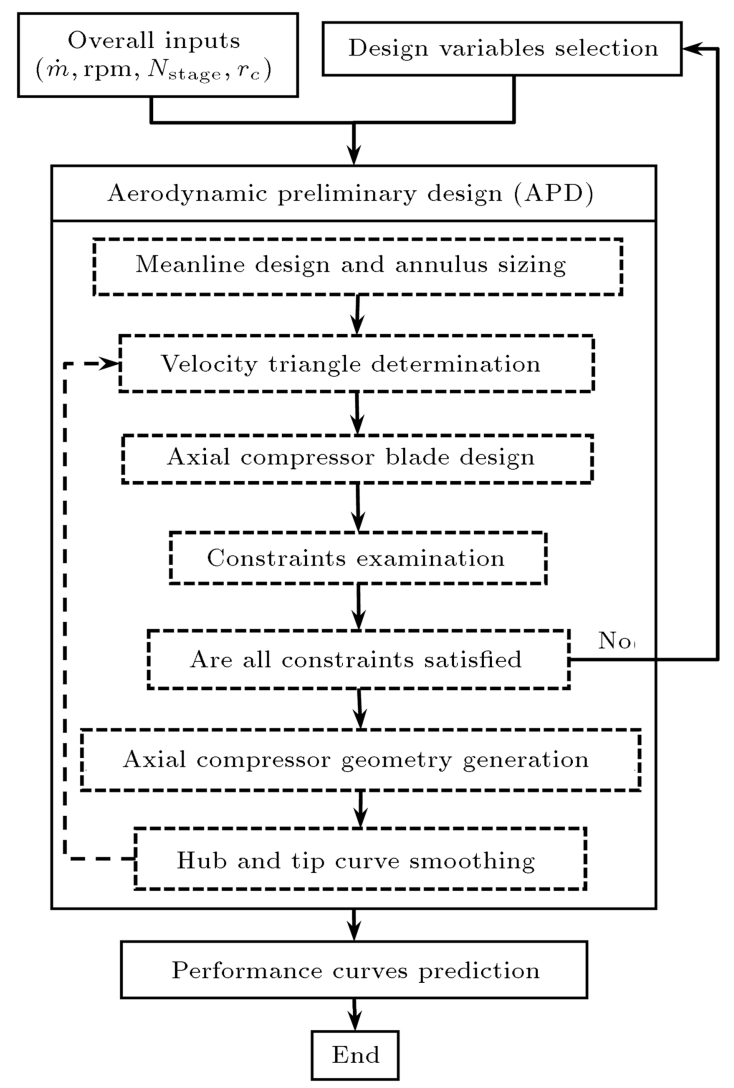

Figure 2. Aerodynamic Preliminary Design and Simulation (APDS) algorithm.

number and mean radius, and aerodynamic design variables. Different designers might select various types of aerodynamic design variables. In some cases, the parameters such as the stagger angle or flow angles are considered as the aerodynamic design variables which are not independent of other variables. The presented algorithm employs the minimum number of independent basic variables, based on which other variables are computed. These variables are classified as the Velocity Triangle Determination (VTD) and Blade Design (BD) ones.

The Aerodynamic Preliminary Design (APD) block utilizes the following modules (Figure 2) [9,10]:

- Meanline design and annulus sizing;

- Velocity triangle determination;

- Blade design;

- Axial compressor three-dimensional geometry generation;

- Hub and tip curve smoothing.

The meanline design and annulus sizing module derive the average pressures in the inlet and outlet stations of the blade rows and, through them, the values of the hub and tip radii of the stations are computed. The velocity triangles are derived on the compressor 
streamlines from the VTD design variables as inputs and the radial equilibrium and vortex equations. The blade design is the next step of algorithm, which uses the blade design variables, obtained velocity triangles, and incidence and deviation angles correlations. Then, a large number of designed constraints are examined and the design variables, in case of having one or more unsatisfied constraints, for one or more stages are modified. These constraints are necessary to satisfy the correlations ranges, to create the suitable blades loading and operating range and also to consider the overall geometry dimensions criteria. If all the constraints are satisfied, the three-dimensional geometry and meridional view of axial compressor will be generated. By use of the generated meridional view of the axial compressor, the hub and tip radii are modified in order to obtain the smooth curves for them. Through the modified radii, the velocity triangle determination, blade design, and geometry generation are iterated until the final design is extracted.

The other block of APDS algorithm is the prediction of performance curves in the design and offdesign conditions, which may be done by the meanline, streamline, or CFD simulator. The meanline and streamline methods (conventional methods) have good accuracy if suitable loss and angles correlations are used. Nowadays, the CFD and conventional simulators are simultaneously employed in the axial compressor design [8]. The preliminary design optimization, which is done by the CFD simulator, is extremely time consuming (from one day to one week, depending on the used computational hardware); therefore, the development and modification of the meanline or streamline methods, due to their low computation time, are still ongoing $[11,12]$. However, it seems that using CFD codes in the future will be common and it may be substituted by meanline or streamlines methods in the optimization problems with the computational hardware advances.

Although the performance curve prediction has major importance in the compressor design, it must be noted that the development of different simulators, with any level of accuracy, does not have any advantage unless they are combined with the APD tool. This is for the generation of the required geometrical inputs of the abovementioned simulators by the APD block. There are many publications available on the developed meanline and streamline simulators $[13,14]$ or the CFD application in the compressor simulation $[15,16]$, but small attention has been paid to the development of the compressor design modules. However, two research works $[17,18]$ have evaluated the effects of some parameters such as solidity, blade angles, or chord on the performance of a single-stage compressor and have optimized these parameters through the meanline method.

In this research, the Aerodynamic Preliminary Design (APD) algorithm has been developed. In the next sections, the required design variables and necessary constraints are explained and the algorithm and computation method of any APD module are described. Finally, the algorithm is employed to redesign the NACA 8-stage transonic compressor [19] by using its design variables. The velocity triangles and blade specifications of the redesigned compressor are compared with those of the NACA 8-stage compressors and the three-dimensional geometry of redesigned compressor is presented.

\section{Design variables}

The aerodynamic preliminary design is done by selecting some design variables. The essential design variables and their number for designing a single-stage compressor are tabulated in Table 1. As seen, a

Table 1. The aerodynamic preliminary design variables.

\begin{tabular}{cllc}
\hline Type & \multicolumn{1}{c}{ Variable } & \multicolumn{1}{c}{ Description } & Number \\
\hline \multirow{4}{*}{ VTD } & $n, m$ & Vortex exponent & 1 \\
& $\phi_{1 c}, \phi_{2 c}^{*}$ & Meanline flow coefficients & 1,1 \\
& $R_{c}$ & Meanline stage reaction & 1 \\
& $\psi_{c}$ & Stage work coefficient & 1 \\
\hline \multirow{4}{*}{ Blade design } & $\sigma_{c}$ & Meanline solidity & 2 \\
& $\left.\frac{t_{\max }}{c}\right|_{\text {hub }},\left.\frac{t_{\max }}{c}\right|_{\text {tip }}$ & Blades hub and tip max. thickness ratio & $2,2^{* *}$ \\
& $F_{t h}$ & Tip to hub chord ratio & 1,1 \\
& AR & Aspect ratio & 2 \\
& $($ DCA profiles $)$ & Leading and trailing edges radii & 4 \\
\hline
\end{tabular}


vast number of design variables must be selected by the designer. For example, the designer must select minimum 150 (all blades are designed with NACA 65 profile) and maximum 190 (all blades are designed with DCA profiles) design variables for designing a 10-stage axial compressor.

Table 1 shows the maximum aerodynamic design variables which can be used in the aerodynamic preliminary design of axial compressors; however, all of them are not employed in the industrial designs due to the following reasons:

- Different vortex types for different stages are not used in the preliminary design and one or two vortex types are usually applied for all stages;

- In some industrial designs, the chord ratios of all the blade rows are considered equal to one and in other designs, the chord variation is applied for the front stages;

- The lower values of leading and trailing edge radii lead to lower profile and shock losses. However, this reduction cannot be lower than the critical value since it leads to blade mechanical damage and deformation. Therefore, the leading and trailing edge radii of blades cannot be determined in the aerodynamic design and they depend on the materials which are used in the blade manufacturing.

According to the above reasons, the design variables number is decreased considerably. For example, the design variables for the aforementioned 10-stage axial compressor with four transonic and variable chord stages and single vortex-type are reduced to 88 .

\section{Design constraints}

The design constraints are divided into aero-dynamical and geometrical ones, which are presented in Table 2. The absolute and relative flow angles and the flow deflection angles are considered to observe the blade design correlations ranges. Diffusion factor is a major parameter in the preliminary design to control the blades loading and losses and has major influences on compressor efficiency, surge margin, and weight. Its

Table 2. Preliminary design constraints.

\begin{tabular}{cc}
\hline Type & Constraints \\
\hline \multirow{2}{*}{ Aerodynamic } & $0 \leq \beta \leq 60$ \\
& $0 \leq \varepsilon \leq 60$ \\
& Diffusion factor \\
& Max. inlet Mach number \\
Geometrical & Compressor length \\
& Max. diameter
\end{tabular}

higher value causes low blade number, weight, and surge margin and its lower value leads to high blade number and weight and also low efficiency. Due to the compressibility effects, the maximum inlet Mach number is dependent on the performance characteristics of the blade profiles. The geometrical constraints are dictated by the gas turbine system designer and they might be one of the compressor maximum diameters and length limits or both of them.

\section{Meanline design and annulus sizing}

The axial velocities in the mean radii of the inlet and outlet blade rows are obtained from the flow coefficient definitions:

$$
\begin{aligned}
& C_{a, 1}=\phi_{c 1} \cdot U_{1}, \\
& C_{a, 2}=\phi_{c 2} \cdot U_{2},
\end{aligned}
$$

and the tangential velocities in these radii are computed through the simplified vortex equations [10]:

$$
\begin{aligned}
& C_{\theta 1}=U_{c 1}\left(1-R_{c}-\frac{\psi_{c}}{2}\right), \\
& C_{\theta 2}=\left(\frac{r_{1}}{r_{2}}\right)\left(C_{\theta 1}+\psi_{c} U_{1}\right), \\
& \psi_{c}=\frac{\Delta H_{c}}{U_{1}^{2}}
\end{aligned}
$$

where $C_{a}$ is the axial velocity, $C_{\theta}$ is the tangential velocity, $\phi_{c}$ is the mean flow coefficient, $\psi_{c}$ is the mean work coefficient, $R_{c}$ is the mean reaction, $U$ is the blade velocity, $r$ is the radius, $\Delta H_{c}$ is total enthalpy increase, and subscripts 1 and 2 indicate the rotors inlet and outlet stations.

The Mach numbers in the inlet and outlet of the blades are computed by the total temperatures, which are derived from the work coefficient definition and the obtained velocity triangles [10]:

$$
\begin{aligned}
& T_{t 2}=T_{t 1}+\frac{\psi_{c} U_{c 1}^{2}}{c_{p}}, \\
& T=T_{t}-\frac{C^{2}}{2 c_{p}}, \\
& M=\frac{C}{\sqrt{\gamma R T}}, \\
& M^{\prime}=\frac{W}{\sqrt{\gamma R T}},
\end{aligned}
$$

where $T_{t}$ is the absolute total temperature, $T$ is the static temperature, $C$ is the absolute velocity, $W$ is the relative velocity, $M$ is the Mach number, and $M^{\prime}$ is the relative Mach number. 
The blades are designed on the meanline radius by using the blade design variables and obtained velocity triangles. Using the Kock and Smith [20] and Aungier [10] loss models, the profile, secondary and end wall losses are obtained and pressures in the inlet and outlet of blades are calculated. Through the computed pressures and mass flow continuity, the necessary annulus area (hub and tip radii) for the design mass flow are computed. These radii are the initial values and are modified by computing the boundary layer displacement thickness. Experiments show that this thickness grows in the flow direction in the compressor, but its value tends to a constant value after several stages $[10,19]$. The computation of the displacement thickness effect is done by the famous meanline Kock [21] method for the first five stages and the other stage thicknesses are considered equal to the one in the 5 th stage.

\section{Velocity triangle determination}

The velocity triangles are determined through Eqs. (10) to $(13)[10]$ :

$$
\begin{aligned}
& C_{a} \frac{\partial C_{a}}{\partial r}+\frac{C_{\theta}}{r} \frac{\partial\left(r C_{\theta}\right)}{\partial r}=\frac{\partial H}{\partial r}-T \frac{\partial s}{\partial r} \\
& \frac{C_{\theta 1}}{U_{C 1}}=\frac{\left(1-R_{c}\right)}{R_{D}^{n}}-\frac{\psi_{c}}{2 R_{D}{ }^{m}} \\
& C_{\theta 2}=C_{\theta 1} \frac{U_{1}}{U_{2}}+\psi \frac{U_{c 1}^{2}}{U_{2}} \\
& R_{D}=\frac{r}{r_{c}}
\end{aligned}
$$

where $H$ is the total enthalpy, $s$ is the entropy, $R_{D}$ is the non-dimensional streamline radius, $n$ and $m$ are the vortex exponents, and the subscript $c$ indicates the meanline section.

Since in a good design, the entropy spanwise variation is small, the reduction in entropy gradient on the right-hand side of the radial equilibrium equation (Eq. (10)) has not made significant errors in the velocity triangles determination [6]. Using this assumption and solving the differential equation of Eq. (10) in the radial direction, the meridional velocity can be obtained as:

$$
C_{a}^{2}(r)=\left(\phi_{c} U_{c}\right)^{2}+2 \int_{r_{c}}^{r}\left(\frac{\partial H}{\partial r}-\frac{C_{\theta}}{r} \frac{\partial\left(r C_{\theta}\right)}{\partial r}\right) d r .
$$

The 5 VTD steps in the inlet and outlet stations of axial compressor rotors are described below:

1. The velocity triangles are determined on streamlines. The initial streamline radii are computed through the constant area of the streamtubes as- sumption and the hub and tip radii, which were determined in the meanline design and annulus sizing step;

2. The absolute tangential and axial velocities are computed on the streamlines by use of the vortex and radial equilibrium equations (Eqs. (10)-(13)). The other variables such as relative tangential velocity and absolute and relative flow angles are obtained through the triangles geometrical relations $[3,10]$;

3. The rotor outlet total temperature is obtained from the work coefficient, inlet total temperature, and blade mean velocity (Eqs. (6)-(9)). The inlet total temperature in the first rotor is equal to the ambient one and for the other rotors, it is equal to the outlet total temperature of the previous stage.

The static temperature and Mach number in the inlet and outlet stations and on the streamlines are derived from gas dynamic relations [3,10];

4. The variation of the static pressure on the streamlines radii is nearly constant and vary linearly, especially in the design point. This has been shown in different published articles and open access reports [22]. By the radial uniformity of static pressure assumption and integral form of mass flow continuity, the mass flow which passes through the inlet and outlet stations is computed as:

$$
\dot{m}=2 \pi \int_{r}^{r_{t}} \rho C_{a} r d r
$$

The static pressures in different stations are considered equal to the one derived from the meanline design in the previous preliminary design step. The new streamlines radii are determined based on the equal mass flow of the streamtubes and total mass flow equal to the computed mass flow (Eq. (15));

5. Steps 2 to 4 are iterated so that the computed mass flow and velocity triangles converge to their final value within the specified error.

\section{Blade design}

The blade design outputs include the following parameters in all blade rows' streamlines:

- Profile family;

- Camber line type;

- Maximum thickness/chord ratio;

- Solidity;

- Blade number;

- Camber angle;

- Stagger angle;

- Leading and trailing edge radii. 
These parameters must be selected in consistence with the flow angles determined in the VTD step. The relations between the aforementioned parameters and flow angles are constructed with the incidence and deviation angle correlations. Different correlations for different blade profiles have been represented during years [23-25]. Some of the incomplete correlations for the conventional profiles are published in the open sources and many of them, especially for the new or transonic blade profile, are confidential due to the considerable experiment expenses which are required for their derivation.

Vast numbers of blade and flow parameters are involved in these correlations, which make it impossible to compute all the blade parameters directly. Therefore, the blade should be designed through the selection of some blade parameters and the computation of the others in a trial and error method. The parameters which are selected in the blade design process include the solidity and maximum thickness/chord ratio on the streamlines and the blade aspect ratio.

The variation of blade thicknesses is computed through selecting a distribution type and the required thickness ratio values in some radii. The hyperbolic distribution is one of the most used distributions, which leads to low blade mass flow and centrifugal stress [19]. By use of this distribution and the thickness ratio in hub and tip sections, the thickness variation in different radii can be derived as:

$$
\begin{aligned}
& \frac{t_{\mathrm{max}}}{c}(r)=\left.\frac{t_{\mathrm{max}}}{c}\right|_{\text {tip }} \\
& \quad+\left(\left.\frac{t_{\mathrm{max}}}{c}\right|_{\mathrm{tip}}-\left.\frac{t_{\mathrm{max}}}{c}\right|_{\mathrm{hub}}\right)^{2}\left(\frac{r_{\mathrm{tip}}-r}{r_{\mathrm{tip}}-r_{\mathrm{hub}}}\right)^{2}
\end{aligned}
$$

where $\frac{t_{\max }}{c}$ is the maximum thickness/chord ratio and the subscripts "hub" and "tip" indicate the blade hub and tip sections.

Different solidity variation relations can be derived if different chord distributions are considered. The radial distribution of the solidity is calculated by Eq. (17), which is the result of the linear chord variation assumption [10]:

$$
\sigma=\sigma_{c} \frac{1+\left(r-r_{\text {hub }}\right)\left(F_{t h}-1\right) /\left(r_{\text {tip }}-r_{\text {hub }}\right)}{1+\left(r_{c}-r_{\text {hub }}\right)\left(F_{t h}-1\right) /\left(r_{\text {tip }}-r_{\text {hub }}\right)} \frac{r_{c}}{r},
$$

where $\sigma$ is solidity and $F_{t h}$ is tip to hub chord ratio.

The blade design algorithm is shown in Figure 3. In addition to the blade design variables, the rotor inlet and outlet flow angles and tip inlet Mach number (the relative Mach number for rotors and the absolute Mach number for stators) are the required inputs of the blade design.

The profile is selected by the Mach number value.

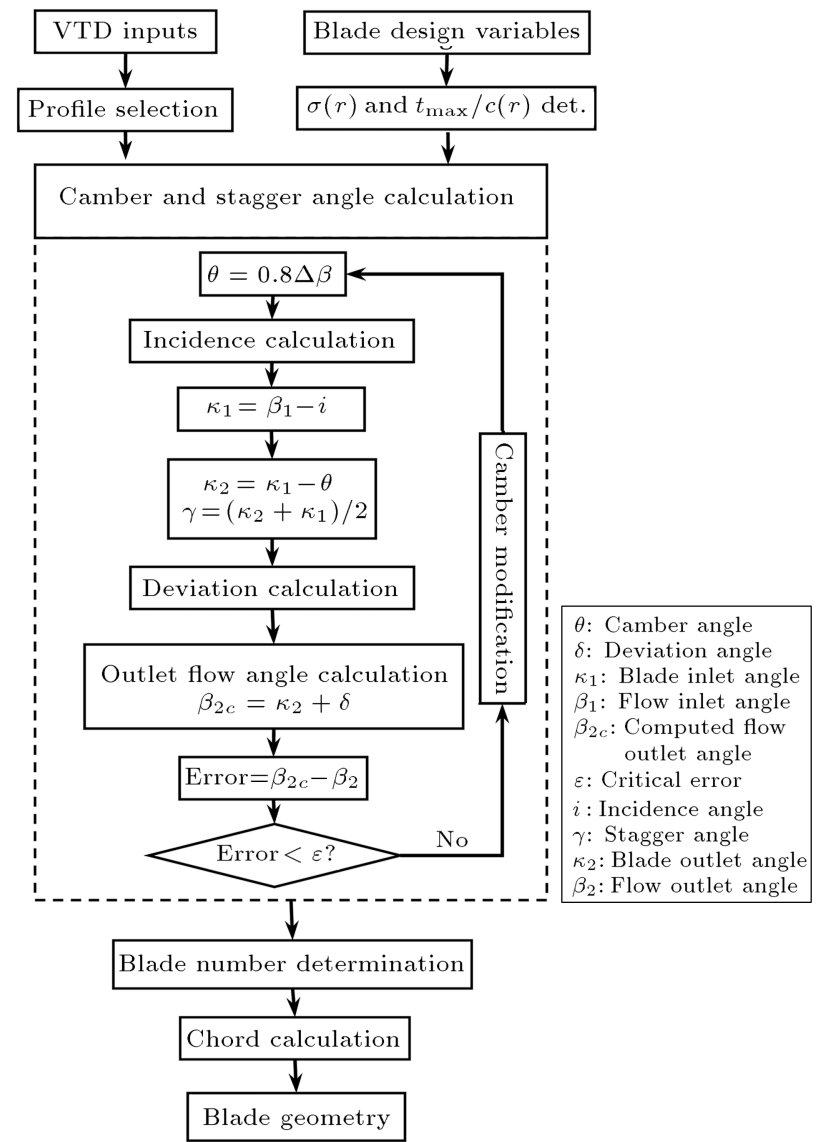

Figure 3. Blade design algorithm.

Cumpsty [26] has mentioned that the NACA 65 profiles can be used for the Mach number up to 0.80 and in the upper values, the DCA blades should be used. The MCA profiles allow designers to have supersonic Mach number up to 1.3. Use of any profiles in the developed algorithm is possible, but the designer must use their special incidence and deviation correlations.

After blade profile selection, the radial distribution of solidity and maximum thickness/chord ratio are determined. The blade camber and stagger angles are calculated using the incidence and deviation angle correlations. This process is identical for all the blade sections in different radii and it is started by the camber angle assumption equal to 80 percent of the flow deflection. The incidence angle is computed and the blade inlet angle is obtained from it and flow inlet angle. The blade outlet and stagger angle are derived by the geometrical relations and the deviation angle is calculated through them. The outlet flow angle is obtained from the computed blade outlet and deviation angles. This angle is compared with the outlet flow angle, which is determined in the VTD step. The camber angle is modified to converge to the computed and VTD outlet flow angles.

The blade number determination is the next step of the blade design and is done using the annulus radii, 
mean solidity, and aspect ratio:

$$
\begin{aligned}
Z & =\sigma_{c} \frac{2 \pi r_{c}}{c_{c}}, \\
c_{c} & =\frac{r_{\mathrm{tip}}-r_{\mathrm{hub}}}{\mathrm{AR}},
\end{aligned}
$$

where $Z$ is the blade number, $c_{c}$ is the mean chord, and $\mathrm{AR}$ is the aspect ratio.

If the calculated blade number is a decimal digit, the nearest integer number will be chosen as the number of blades. Finally, the chord magnitudes in different radii can be calculated by the solidity distribution and blade number:

$$
c=\sigma \frac{2 \pi r}{Z} \text {. }
$$

\section{Axial compressor geometry generation}

The output of the preliminary design is the threedimensional geometry of the blades which will be used in the other compressor design steps such as the mechanical analysis and the detailed design (Figure 1). The geometrical outputs of the meanline design and annulus sizing, VTD, and blade design modules are the streamlines radii and blade specifications (i.e. the camber and stagger angles, the maximum thickness/chord ratio, and the leading and trailing edges radii) on the streamlines. These data cannot be used in other design steps and they should be changed to the points and nodes coordinates of the blades and annulus geometry. For this reason, a code has been developed which generates the three-dimensional blades geometry and assembles them over the compressor axis. This code produces the blade section profiles coordinates on the streamline and stacks them through a guideline curve which connects the area centroids of the blade sections (Figure 4). Figures 5 and 6 show the generated

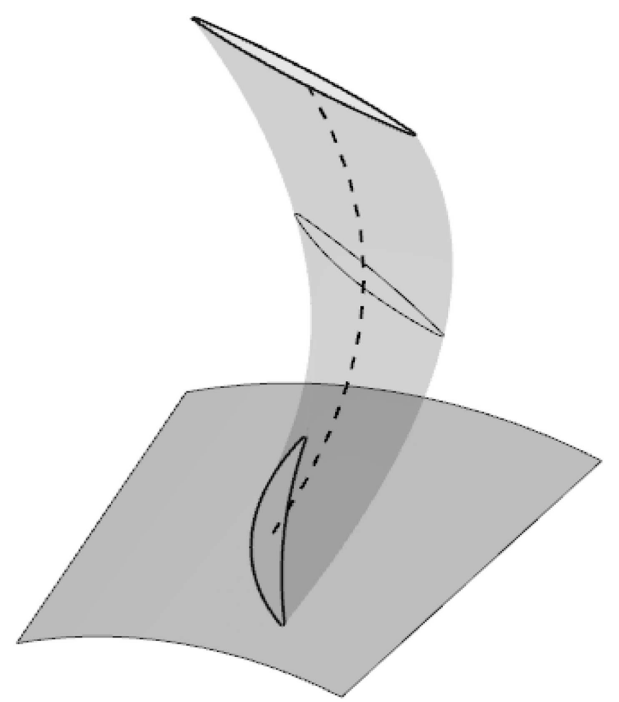

Figure 4. Blade sections and guideline curve.

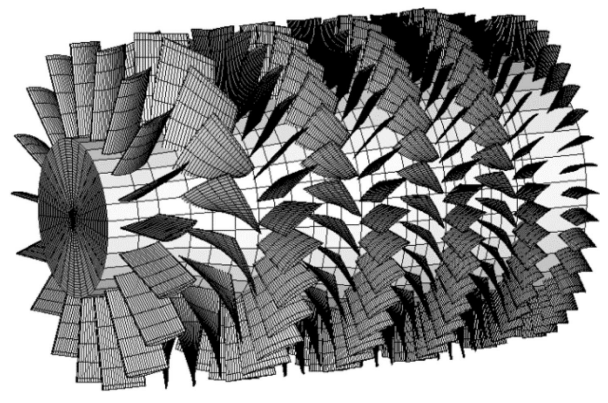

Figure 5. BBC-Sulzer compressor geometry.

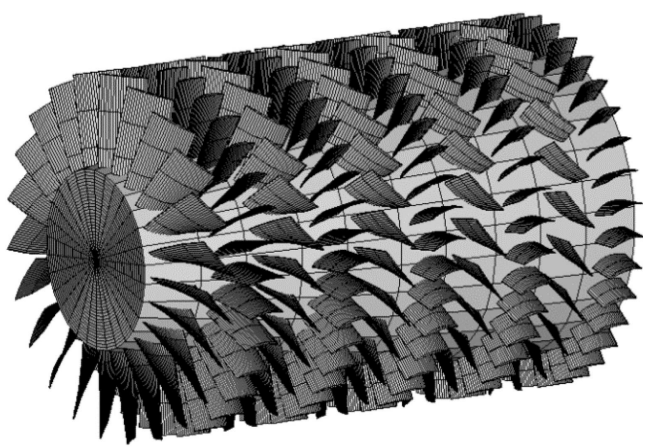

Figure 6. NACA 5-stage compressor geometry.

geometry of BBC/Sulzer compressor [22] and NACA 5-stage compressor [27] from the developed code.

These figures are plotted in the Tecplot software. However, the developed code can generate the input files proper for the numerical software such as ANSYS CFX and modeling software such as UNG NX. These files considerably reduce the modeling time of the axial compressor in the software. In addition, some mechanical specifications such as the blades mass and sections centrifugal stress can accurately be computed. The volumes are compared in Tables 3-5; also, centrifugal forces of BBC-Sulzer blades, which have been derived from the developed code, have been compared with those from UNG NX and ANYSY software.

\section{Algorithm evaluation}

The APD algorithm is applied to a computer code which has been developed with FORTRAN programming language. In this section, the results of the developed code for the NACA 8-stage transonic axial

Table 3. Rotors' volume of BBC-Sulzer compressor $\left(\mathrm{mm}^{3}\right)$.

\begin{tabular}{cccc}
\hline Stage & Code & SIMENSE NX & $\begin{array}{c}\text { Difference } \\
(\%)\end{array}$ \\
\hline 1 & 67857.88 & 67725.25 & 0.20 \\
2 & 22925.23 & 22896.16 & 0.13 \\
3 & 14872.78 & 14822.54 & 0.34 \\
4 & 10517.83 & 10473.63 & 0.42 \\
\hline
\end{tabular}


Table 4. Stationary blades' volume of BBC-Sulzer compressor $\left(\mathrm{mm}^{3}\right)$.

\begin{tabular}{|c|c|c|c|}
\hline $\begin{array}{c}\text { Blade } \\
\text { type }\end{array}$ & Code & Ansys & $\begin{array}{c}\text { Difference } \\
(\%)\end{array}$ \\
\hline IGV & 63869.01 & 64142.45 & -0.43 \\
\hline OGV & 7461.83 & 7460.12 & 0.02 \\
\hline \multirow{4}{*}{ 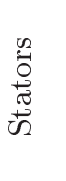 } & 33170 & 33179.61 & -0.03 \\
\hline & 15296.13 & 15288.04 & 0.05 \\
\hline & 9863.59 & 9857.41 & 0.06 \\
\hline & 6441.17 & 6443.20 & -0.05 \\
\hline
\end{tabular}

Table 5. Rotors' root centrifugal force of BBC-Sulzer compressor $(\mathrm{N})$.

\begin{tabular}{cccc}
\hline Stage & Code & Ansys & Difference (\%) \\
\hline 1 & 248969.80 & 248810 & 0.06 \\
2 & 95113.61 & 95095 & 0.02 \\
3 & 66216.72 & 66173 & 0.07 \\
4 & 46868 & 46834 & 0.07 \\
\hline
\end{tabular}

Table 6. VTD design parameters.

\begin{tabular}{cccccccc}
\hline Stage & $\boldsymbol{\psi}_{\boldsymbol{c}}$ & $\boldsymbol{\phi}_{\boldsymbol{1} \boldsymbol{c}}$ & $\boldsymbol{\phi}_{\boldsymbol{2 c}}$ & $\boldsymbol{R}_{\boldsymbol{c}}$ & $\boldsymbol{n}$ & $\boldsymbol{m}$ & $\boldsymbol{\eta}$ \\
\hline 1 & 0.800 & 0.588 & 0.633 & 0.400 & +1 & +1 & 0.88 \\
2 & 0.620 & 0.569 & 0.603 & 0.398 & 0 & 0 & 0.88 \\
3 & 0.503 & 0.565 & 0.572 & 0.332 & -1 & -1 & 0.91 \\
4 & 0.486 & 0.557 & 0.562 & 0.371 & -1 & -1 & 0.91 \\
5 & 0.478 & 0.605 & 0.558 & 0.400 & -1 & -1 & 0.91 \\
6 & 0.473 & 0.560 & 0.560 & 0.432 & -1 & -1 & 0.91 \\
7 & 0.491 & 0.556 & 0.559 & 0.447 & -1 & -1 & 0.91 \\
8 & 0.486 & 0.545 & 0.548 & 0.455 & -1 & -1 & 0.88 \\
Virtual & 0.486 & 0.545 & 0.548 & 0.455 & -1 & -1 & 0.85 \\
\hline
\end{tabular}

compressor [19], which is called NACA compressor in the rest of the article, as well as the rpm of 13380 and mass flow of $29.5(\mathrm{~kg} / \mathrm{s})$ are evaluated. The design variables of NACA compressor were used as the inputs of the developed code. The VTD and BD design variables of NACA compressor are tabulated in Tables 6 and 7 , respectively.
In addition, the following rules have been used in the NACA compressor design:

1. Because of reasons unknown to the authors of the present paper, the velocity triangles in the outlets of rotors 3 to 8 are the average of those obtained through the radial equilibrium equation and the straight-throughflow method. In the straightthroughflow method, the axial velocity in the outlet rotor on any streamline is the average of the stage inlet and outlet:

$$
\begin{aligned}
& C_{a 2}^{i}=0.5\left(C_{a 2, \mathrm{REE}}^{i}+C_{a 2, \mathrm{STF}}^{i}\right), \\
& C_{a 2, \mathrm{STF}}^{i}=\left(C_{a 1, \mathrm{REE}}^{i}+C_{z 1, \mathrm{REE}}^{i+1}\right) / 2,
\end{aligned}
$$

where $i$ is stage number, REE indicates radial equilibrium equation, and STF indicates straightthrough flow method;

2. The first and second rotors have been designed by the DCA profiles. The constant incidence angle of 4.0 (deg) (Eq. (23)) and deviation angle correlation of Eq. (24) have been used to design these blades:

$$
\begin{aligned}
& i=4, \\
& \delta=0.475 \frac{\theta}{\sigma} .
\end{aligned}
$$

The other blades have been designed by the NACA 65 profiles through Herrig et al. [23] angle correlations.

By employing the abovementioned rules in the VTD and blade design modules and using the design variables of Tables 6 and 7, the NACA 8-stage compressor has been redesigned through the developed APD code. The comparison of the computed velocity triangles of the redesigned compressor with those of NACA compressors is shown in Figure 7.

Except for the velocity triangles values of rotor No. 2, which are different from those obtained by the developed code, the other rotors' velocity triangles

\begin{tabular}{|c|c|c|c|c|c|c|c|c|c|c|}
\hline \multirow{2}{*}{ Stage } & \multicolumn{2}{|c|}{ AR } & \multicolumn{2}{|c|}{$\sigma_{c}$} & \multicolumn{2}{|c|}{$F_{t h}$} & \multicolumn{2}{|c|}{$\left.\frac{t_{\max }}{c}\right|_{\text {hub }}$} & \multicolumn{2}{|c|}{$\left.\frac{t_{\max }}{c}\right|_{\text {tip }}$} \\
\hline & Rotor & Stator & Rotor & Stator & Rotor & Stator & Rotor & Stator & Rotor & Stator \\
\hline 1 & 1.47 & 2.23 & 1.345 & 1.036 & 1.25 & 1 & 0.095 & 0.06 & 0.05 & 0.06 \\
\hline 2 & 1.15 & 1.77 & 1.392 & 1.2 & 1.25 & 1 & 0.086 & 0.06 & 0.048 & 0.06 \\
\hline 3 & 2.19 & 1.99 & 1.134 & 0.897 & 1 & 1 & 0.10 & 0.06 & 0.06 & 0.06 \\
\hline 4 & 1.85 & 1.68 & 1.275 & 0.898 & 1 & 1 & 0.10 & 0.06 & 0.06 & 0.06 \\
\hline 5 & 1.56 & 1.39 & 1.294 & 0.948 & 1 & 1 & 0.08 & 0.06 & 0.06 & 0.06 \\
\hline 6 & 1.31 & 1.17 & 1.232 & 0.979 & 1 & 1 & 0.08 & 0.06 & 0.06 & 0.06 \\
\hline 7 & 1.09 & 0.98 & 1.205 & 1 & 1 & 1 & 0.08 & 0.06 & 0.06 & 0.06 \\
\hline 8 & 0.94 & 0.87 & 1.184 & 1.014 & 1 & 1 & 0.08 & 0.06 & 0.06 & 0.06 \\
\hline
\end{tabular}
have a good conformity with the NACA compressor. The differences in velocity triangles of rotor No. 2

Table 7. Blades design parameters. 

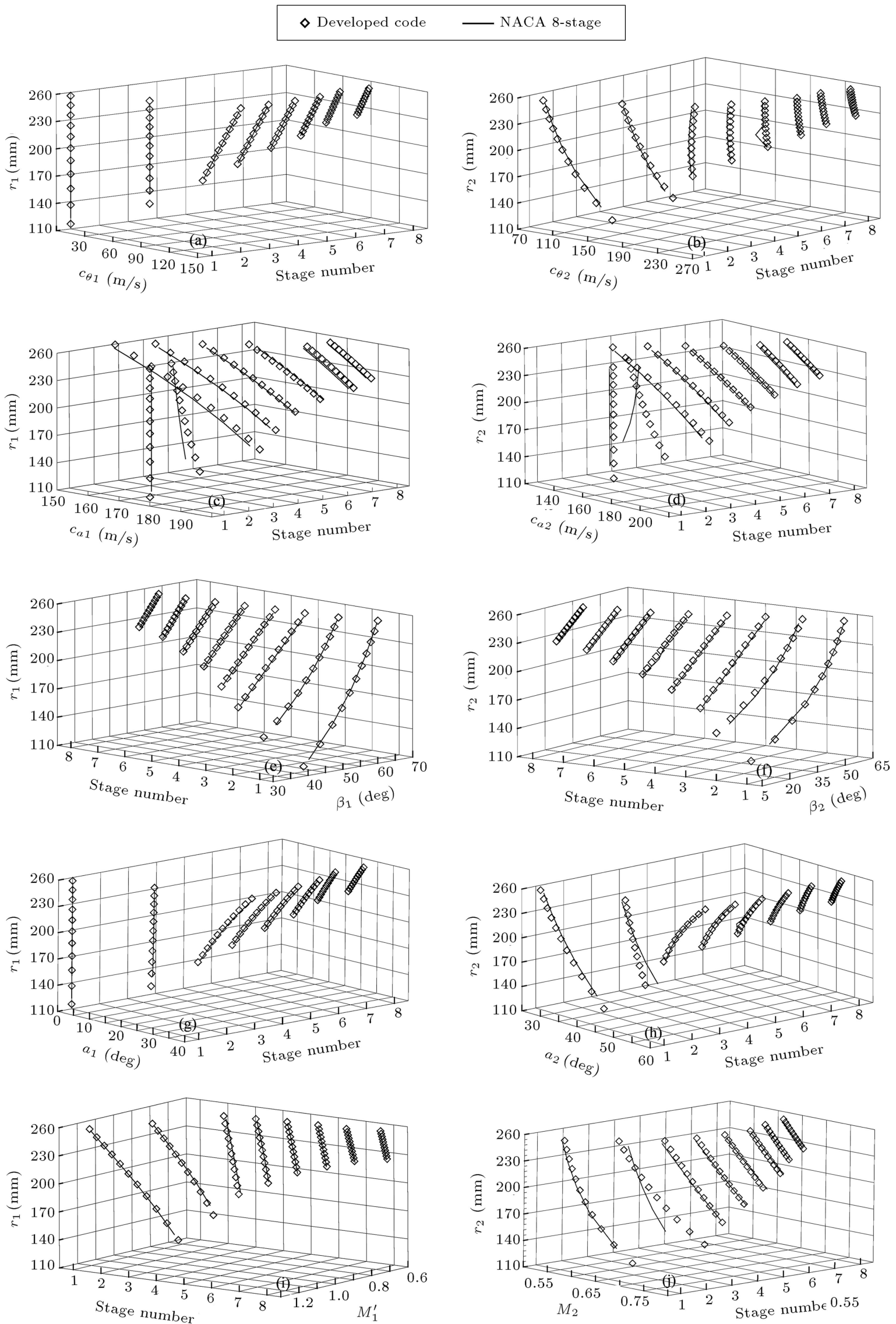

Figure 7. Comparison of the computed velocity triangles with those of NACA compressor. 
are due to the difference in the values and trends of the axial velocities in the redesigned and NACA compressors, the reason of which is unknown to the writers. The surprising point is that the tangential velocities in the inlet and outlet of this rotor are equal in the redesigned and NACA compressors. Based on the radial equilibrium equation, the axial velocity reported for the NACA compressor should be the same as that obtained through the code. Since the axial velocity value and distribution in the other blades rows of NACA compressor are the same as the ones resulted from the code, the VTD module is valid and the probable code errors are not the reason behind the differences of rotor No. 2. In addition, the analytical computation for this rotor shows similar values and trends to the code results. For this reason, the writers guess the reported values for rotor No. 2 may have been wrong or other additional assumptions may have been applied for this rotor, which have not been mentioned in [19].

The NACA compressor velocity triangles have been derived on the axial sections, whereas in the developed code, the streamlines radius variations are incorporated in the VTD. This difference in the computation methods leads to some small differences of velocity triangles between the redesigned and NACA compressors (except for rotor No. 2) (Figure 7).

Figure 8 compares the derived blades camber and stagger angles from the APD code with the ones of NACA compressor. As seen, good conformity in values and trends exists between the rotors blade specifications of NACA compressor and the computed ones (Figure 8(a) and (c)). The computed stators stagger angles have good conformity with the NACA compressor; however, this conformity is not seen in all the stators camber angles. The difference in these values results from the difference between the flow angles and solidity distributions of the code and NACA compressor. In addition, the NACA compressor stators No. 5-8 have been designed with constant values, while this assumption has not been used in the APD code.

The meridional view of NACA compressor is compared with that of the redesigned compressor in Figure 9. Differences are seen in the hub and tip radii of these compressors. These differences are due to the different annulus sizing methods which have been applied for the NACA compressor and the developed code. The annulus sizing for the NACA compressor is done by the assumption of total pressure uniformity in the radial direction. The total pressure was computed through the selection of stages and rotors isentropic

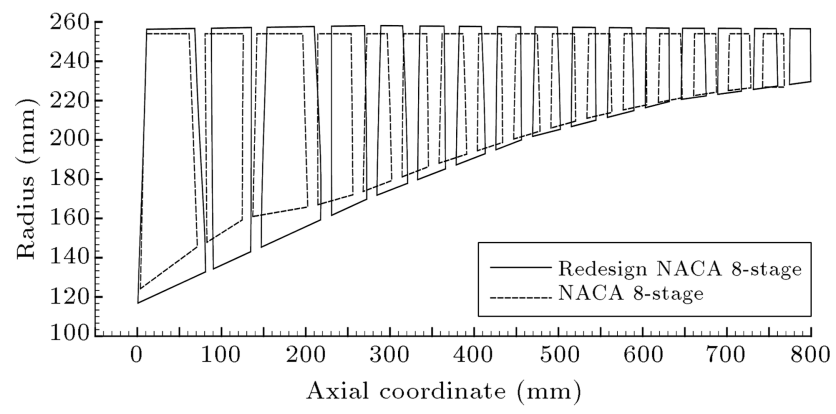

Figure 9. Meridional view comparison of the redesigned and NACA compressors.

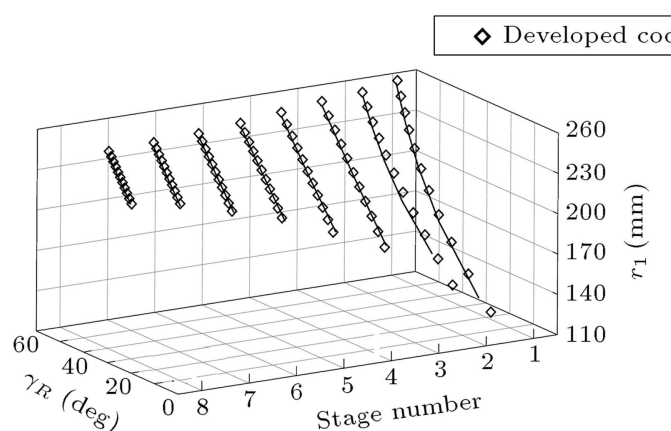

(a)

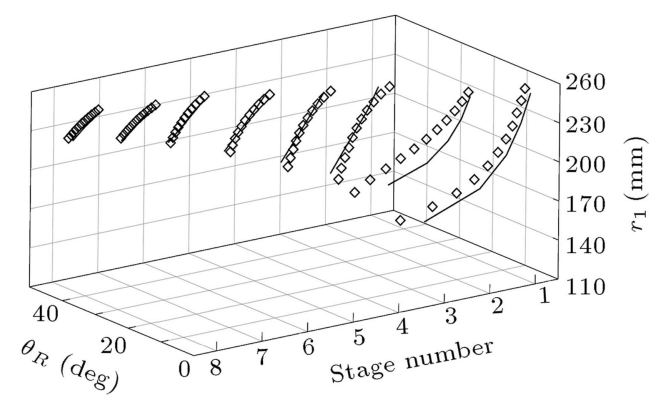

(c)

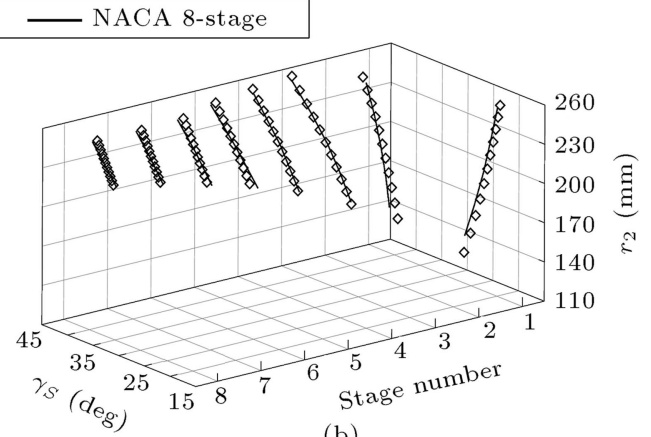

(b)

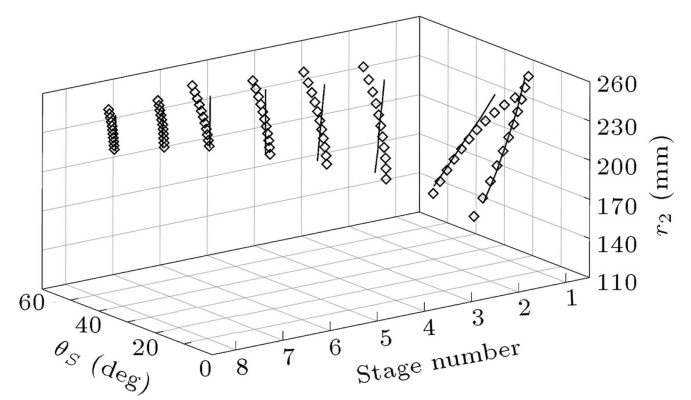

(d)

Figure 8. Comparison of the computed blade specifications with those of NACA compressor. 
efficiency as input. The applied method is completely different from the used method in this article. It must be noted that the assumption of the total pressure uniformity is in contrast with the flow physics [22]; however, the static pressure uniformity used in the developed code satisfies the flow patterns [22]. In addition, the determination of the stage efficiency as input requires to be highly experienced in the axial compressor designing; however, the used meanline design in developed code can derive the static pressure in any station through the empirical loss correlations.

The blades axial coordinates are also different in NACA and redesigned compressors. It is because of different chord values of the compressors' blades. As mentioned, the chord magnitude is dependent on the aspect ratio and blade height (Eq. (19)). Since the blades aspect ratio of the redesigned compressor is equal to that of the NACA compressor, the chord difference is the result of the difference between the compressors hub and tip radii, which leads to lower overall length, of about $30 \mathrm{~mm}$, of NACA compressor than that of the redesigned one.

The three-dimensional geometry of the redesigned compressor is shown in Figure 10. This geometry is the final outcome of the aerodynamic preliminary design algorithm, which is generated through the threedimensional geometry generation module and plot by the Tecplot. However, the required input files for the Computational Fluid Dynamics (CFD) software such as CFX and the mechanical and modeling software such as UNG NX can be generated. The blades sections are generated by the 58 points on their suction and pressure surfaces and 7 points for the leading or trailing edges. These numbers of points result good quality surfaces. The quality of the geometry surfaces and points numbers are shown in Figure 11 for the first rotor.

The computation of the weight and centrifugal stresses is the other ability of the developed geometrical modules. Tables 8 and 9 represent these parameters for the redesigned compressor and compare them with the NACA ones. As seen, the blade weights of

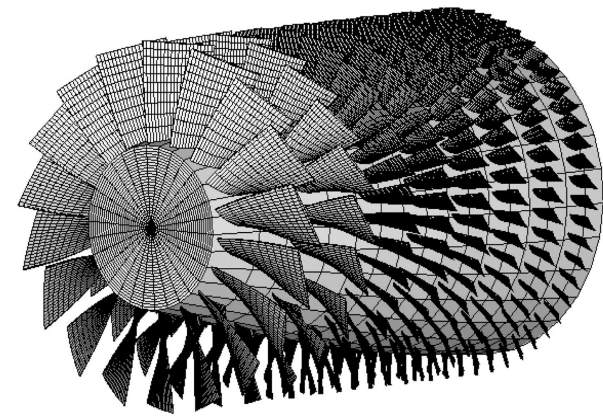

Figure 10. Three-dimensional geometry of the redesigned compressor.

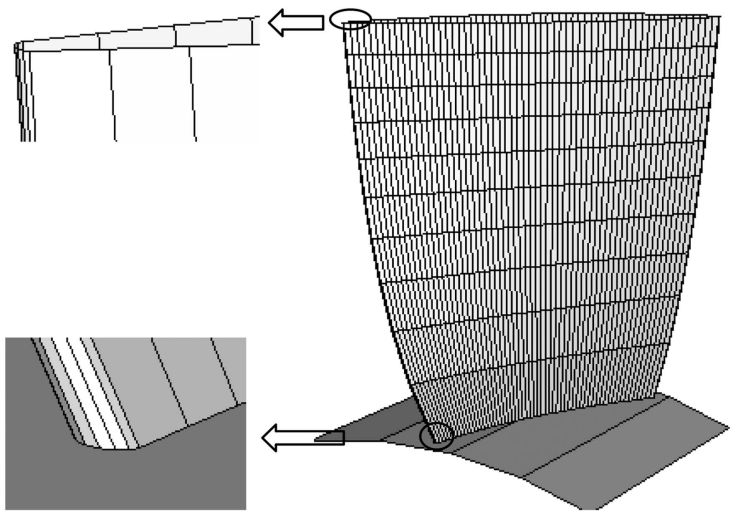

Figure 11. Three-dimensional geometry of the first rotor.

Table 8. Blades weight comparison of redesigned and NACA compressors.

\begin{tabular}{cccccc}
\hline \multirow{2}{*}{ Stage } & \multicolumn{2}{c}{ Rotors } & & \multicolumn{2}{c}{ Stators } \\
\cline { 2 - 3 } \cline { 5 - 6 } & NACA & Redesigned & & NACA & Redesigned \\
\hline 1 & 6.56 & 7.71 & & 2.03 & 2.59 \\
2 & 4.60 & 5.23 & & 2.12 & 2.40 \\
3 & 1.86 & 2.03 & & 1.02 & 1.19 \\
4 & 1.73 & 1.84 & & 0.84 & 0.92 \\
5 & 1.24 & 1.33 & & 0.71 & 0.78 \\
6 & 0.93 & 1.01 & & 0.58 & 0.63 \\
7 & 0.73 & 0.77 & & 0.48 & 0.54 \\
8 & 0.59 & 0.65 & & 0.41 & 0.42 \\
Total & 18.24 & 20.57 & & 8.19 & 9.47 \\
\hline
\end{tabular}

Table 9. Rotors root centrifugal stress comparison of redesigned and NACA compressors ( $\mathrm{MPa})$.

\begin{tabular}{ccc}
\hline Stage & NACA & Redesigned \\
\hline 1 & 325.92 & 326.82 \\
2 & 263.91 & 260.77 \\
3 & 204.99 & 202.48 \\
4 & 173.68 & 168.91 \\
5 & 159.28 & 159.13 \\
6 & 131.00 & 132.28 \\
7 & 108.31 & 108.18 \\
8 & 90.98 & 92.65 \\
\hline
\end{tabular}

the redesigned compressor are heavier than those of the NACA compressor and the redesigned compressor weight is $12(\%)$ larger than the NACA compressor weight. This shows the annulus sizing importance in the compressor weight in addition to its effect on the aerodynamic and performance curves. The rotors centrifugal stresses in the root section of the aforementioned compressors are compared in Table 8. This table shows the same centrifugal stress in two compressors, which is due to increasing root section areas of the redesigned compressor. 


\section{Conclusion}

The Aerodynamic Preliminary Design (APD) is a major step in the axial compressor design because of the high dependency of the final design on its results. In this article, a preliminary design algorithm has been developed. The different steps of the preliminary design, the required design variables, and the design constraints have been introduced. The algorithm is applied to a computer code through FORTRAN programming language and its results have been evaluated through the NACA 8-stage compressor. By developing a geometry generation code, the compressor blade geometries and its meridional view, which can be used in other designing and analysis steps, can be derived.

\section{References}

1. Larosilierw, L.M., Wood, J.R., Hathaway, M.D., Medd, A.J. and Dang, T.Q. "Aerodynamic design study of advanced multistage axial compressor", Report ARL-TR-2859, NASA (2002).

2. Turner, M.G., Merchant, A. and Bruna, D. "Applications of a turbomachinery design tool for compressors and turbines", 43rd AIAA/ASME/SAE/ASEE Joint Propul. Conf. and Exhib., Cincinnati, OH, USA (2007).

3. Saravanamuttoo, H.I.H., Rogers, G.F.C., Cohen, H. and Straznicky, P., Gas Turbine Theory, 6th Edn., Pearson Education, Canada (2008).

4. Okui, H., Verstraete, T., Braembussche, R.A. and Alsalihi, Z. "Three-dimensional design and optimization of a transonic rotor in axial flow compressors", $J$. of Turbomach., $\mathbf{1 3 5}(3)$ (2013).

5. Oyama, A., Liou, M. and Obayash, S. "Transonic axial flow blade optimization: evolutionary algorithm/three-dimensional Navier-Stocks solver", $J$. of Propul. and Power, 20(4), pp. 612-619 (2004).

6. Tanimura, K., Murakami, N., Matsuoka, A., Ishida, K., Kato, H., Sakai, T., Taniguchi, T. and Taki, H. "Development of an 8MW-class high-efficiency gas turbine, M7A-03", Proc. of ASME Turbo Expo: Power for Land, Sea and Air, Montreal, Canada (2007).

7. Wadia, A.R., Wolf, D.P. and Haaser, F.G. "Aerodynamic design and testing of an axial flow compressor with pressure ratio of 23.3:1 for the LM25001 gas turbine", J. of Turbomach., 124(3), pp. 331-340 (2002).

8. Ikeguchi, T., Matsuoka, A., Sakai, Y. and Sakano, Y. "Design and development of a 14-stage axial compressor for industrial gas turbine", Proc. of ASME Turbo Expo: Turbine Tech. Conf. and Exp., Copenhagen, Denmark, pp. 125-134 (2012).

9. Smith, H.L. "Axial compressor aero design evolution at general electric", J. of Turbomach., 124(3), pp. 321330 (2002).

10. Aungier, R.H., Axial Flow Compressors: A Strategy for Aerodynamic Design and Analysis, ASME Press, New York, USA (2003).
11. Boyer, K.M. and O'Brien, W.F. "An improved streamline curvature approach for off-design analysis of transonic axial compression systems", J. of Turbomach., 125(3), pp. 475-481 (2003).

12. Templalexis, I., Pilidis, P., Pachidis, V. and Kotsiopoulos, P. "Development of a two dimensional streamline curvature code", J. of Turbomach., 133(1) (2010).

13. Shadaram, A., Fathi, A. and Azizi, R. "Optimization of variable stator's angle for off design compression system using streamline curvature method", ASME Turbo Expo.: Power for Land, Sea, and Air, 7, Orlando, USA, pp. 525-532 (2009).

14. Madadi, A. and Benisi, A.H. "Performance predicting modeling of axial flow compressor at design and offdesign conditions", ASME Turbo. Expo.: Power for Land, Sea, and Air, 6, Berlin, Germany, pp. 317-324 (2008).

15. Fritsch, G., Hoeger, M., Blaha, C. and Bauer, D. "Viscous three-dimensional simulation of transonic compressor stage on parallel hardware", J. of Propul. and Power, 16(3), pp. 388-396 (2000).

16. Brandvik, T. and Pullan, G. "An accelerated 3D Navier-Stokes solver for flows in turbomachines", $J$. of Turbomach., 133(2) (2010).

17. Chen, L., Sun, F. and Wu, C. "Optimum design of a subsonic axial-flow compressor stage", Appl. Energy, 80(2), pp. 187-195 (2005).

18. Chena, L., Luoa, J., Suna, F. and Wub, C. "Design efficiency optimization of one-dimensional multi-stage axial-flow compressor", Appl. Energy, 85(7), pp. 625633 (2008).

19. Voit, H.C. "Investigation of a high pressure ratio eight stage axial flow research compressor with two transonic inlet stage I- aerodynamic design", Report NACA-RME53124, Lewis Flight Propul. Lab., Cleveland, Ohio, USA (1953).

20. Koch, C.C. and Smith, L.H. "Loss sources and magnitudes in axial-flow compressors", J. of Eng. Gas Turbines and Power, 98(3), pp. 411-424 (1976).

21. Koch, C.C. "Stalling pressure rise capability of axial flow compressor stages", J. of Eng. Gas Turbines and Power, 103(4), pp. 645-656 (1981).

22. Hirsh, C. and Denton, J.D. "Through flow calculations in axial turbomachines", Report AGARD-AR175 (1981).

23. Herrig, L.J., Emery, J.C. and Erwin, J.R. "Systematic two-dimensional cascade tests of NACA 65 -series compressor blades at low-speeds", Report NACA-TN9916, Langley Aero. Lab. (1951).

24. König, W.M., Hennecke, D.K. and Fottner, L. "Improved blade profile loss and deviation angle models for advanced transonic compressor blading: Part I-a model for subsonic flow", J. of Turbomach., 118(1), pp. $73-80$ (1996)

25. Banjac, M., Petrovic, M.V. and Wiedermann, A. "A new loss and deviation model for axial compressor inlet guide vanes", J. of Turbomach., 136(7) (2014). 
26. Cumpsty, N.A., Compressor Aerodynamics, Longman Scientific and Technical, UK, pp. 46-129 (1989).

27. Sandercock, M.D., Kovach, K. and Lieblein, S. "Experimental investigation of a 5 -stage axial flow research compressor with transonic rotors in all stages I- compressor design", Report NACA-RM-E54F24, Lewis Flight Propul. Lab., Cleveland, Ohio, USA (1954).

\section{Biographies}

Rohollah Azizi is a $\mathrm{PhD}$ candidate in Aerospace Engineering at K. N. Toosi University. He received his BSc degree from Shahrekord University in Mechanical Engineering and his MSc degree from K. N. Toosi University in Energy Conversion. He has been doing research for more than 7 years on axial compressor simulation and design and has managed to develop several aero-dynamical and thermos-dynamical codes. He has cooperated with some Iranian turbomachinery companies such as TUGA, Turbotec, Monenco, and Tadbirgaran.
Reza Ebrahimi is an Associate Professor and now is the head of Aerospace Engineering Faculty of KNTOOSI University. He received his $\mathrm{PhD}$ and $\mathrm{MSc}$ degrees from Sharif University in Thermal Science. His major interests are reactive flow, propulsion (gas turbines and rockets), CI and SI engines simulation, heat and mass transfer, rarified gas dynamic, and energy conversion systems. He has published several articles in different journals and has cooperated in different industrial projects.

Masoud Ziabasharhagh is an Associate Professor at K.N. Toosi University. He was the Head of Mechanical Engineering Faculty for several years. He has received his $\mathrm{PhD}$ degree from Cranfield University and his MSc degree from Birmingham University in Energy Conversion. His major interests are power plant, thermodynamic, and turbomachinery. He has published several articles in various journals and won the second place in Khwarizmi Festival for constructing steam power plant deaerator. 